\title{
Separación de las clases de lípidos neutros de polen apícola mediante cromatografía líquida de alta resolución (HPLC)
}

\author{
Por S. Muniategui ${ }^{*}$ M. T. Sancho, J. López, J. F. Huidobro y J. Simal. \\ Departamento de Química Analítica, Nutrición y Bromatología. \\ Area de Nutrición y Bromatología. Facultad de Farmacia. Universidad de Santiago de Compostela. \\ 15706 Santiago de Compostela (España)
}

\section{RESUMEN}

Separación de las clases de lípidos neutros del polen apicola mediante cromatografía liquida de alta resolución (HPLC).

Se describe un método rápido mediante HPLC para la separación de las diferentes clases de lípidos neutros del polen apícola en condiciones isocráticas, con detección UV a $206 \mathrm{~nm}$ e inyección directa de la muestra en columna de sílica. Como fase móvil se usa n-hexano-2-propanol-ácido acético $(100: 0,5: 0,1)$. Se aislaron 3 fracciones de lípidos neutros en las 35 muestras analizadas: Esteres de esteroles y otros componentes no polares tales como carotenoides y ceras; triglicéridos y ácidos grasos.

PALABRAS-CLAVE: Cromatografía líquida de alta eficacia - Detección UV - Lípidos neutros (separación) - Polen apicola.

\section{SUMMARY}

Separation of neutral lipid classes from bee-collected pollen by high performance liquid chromatography (HPLC).

A fast method for the separation of neutral lipid classes of bee-collected pollen by isocratic HPLC is described using ultraviolet detection at $206 \mathrm{~nm}$ and direct injection of the sample into a silica column. The mobile phase was $n$-hexane-2-propanolacetic acid (100:0.5:0.1). Three fractions of neutral lipids were isolated from 35 samples: Sterol esters and other nonpolar compounds such as carotenoids and waxes; triglycerides; and fatty acids.

KEY-WORDS: Bee-collected pollen - High performance liquid chromatography - Neutral lipids (separation) - UV detection.

\section{INTRODUCCION.}

En la separación de las distintas clases de lípidos del polen apícola, empleando la cromatografía en capa fina (TLC), se han aislado diferentes fracciones como: Fosfolípidos, esteroles, ácidos grasos, triglicéridos, ésteres de esteroles y ceras (1).
A pesar de las ventajas y posibilidades de la TLC, esta técnica presenta inconvenientes en la precisión y en la reproducibilidad de la medida, además de necesitar para la cuantitativa, el empleo de técnicas como densitometrías y otros métodos que precisan una extracción previa de los componentes como la GLC y HPLC. Por lo cual, en este trabajo se ha preferido emplear la cromatografía líquida de alta resolución, por su mayor precisión y rapidez.

Al no hallarse referencias bibliográficas donde se aplique esta técnica al estudio de los lípidos neutros del polen apícola, se han considerado las descritas para otros tipos de muestras (tejidos animales, cereales y otros).

En la identificación de los lípidos mediante HPLC se utilizan distintos detectores. El más frecuente es el detector UV, operando a longitudes de onda de 206 $\mathrm{nm}$ (2) (3), $213 \mathrm{~nm}$ (4) y $220 \mathrm{~nm}$ (5); también son utilizados el detector de índice de refracción (6) (7), el detector de masas (8) y diode array (5).

En la mayoría de los casos la determinación se realiza con una fase móvil constante en condiciones isocráticas, aunque la aplicación de un gradiente de elución es utilizada en el caso de la separación de lípidos polares y apolares en un mismo cromatograma (5) (8) (9) (10).

Las columnas empleadas son generalmente de sílica, trabajando a temperatura ambiente y en ciertas ocasiones se utiliza un gradiente de flujo (2) (4) (5) para aumentar la resolución y la rapidez del análisis. Algunos autores (3) (6) eliminan previamente los componentes polares (fosfolípidos) por adsorción en ácido silícico antes de inyectar las muestras al cromatógrafo.

En este trabajo se describe un método rápido para determinar la fracción de lípidos neutros del polen apícola comercial mediante HPLC, con inyección directa de la muestra en condiciones isocráticas. 


\section{PARTE EXPERIMENTAL.}

\subsection{Muestras.}

El estudio se ha realizado sobre 35 muestras de polen apícola comercial. Las muestras analizadas incluyen pólenes de las familias Cistaceae 43,9\% (Cistus ladaníferus L. 39,5\%); Borraginaceae (Echium sp. 10,3\%); Compositae 9,3\%; Fagaceae 8,6\% (Quercus sp. 6,8 \%) y en menor cuantía Rosaceae, Campanulaceae, Papilonaceae, Cruciferae, Papaveraceae, Ericaceae y otros.

Con el fin de facilitar las determinaciones es necesario emplear las muestras pulverizadas. La fragmentación se realiza en una atmósfera inerte y exenta de humedad durante uno a dos minutos (11) (12).

\subsection{Extracción de la grasa.}

Se realiza según el análisis de la grasa total en chocolates norma UNE 34-082-76 (13). En el desarrollo del método se distinguen tres etapas: horas.

a) desecación de la muestra a $60^{\circ} \mathrm{C}$ durante 12

b) hidrólisis clorhídrica para liberar los componentes lipídicos ligados.

c) extracción de los lípidos en Soxhlet, durante 4 horas con éter de petróleo.

\subsection{Cromatagrafía líquida de alta resolución.}

\section{Reactivos.}

Solventes calidad HPLC Lichrosolv Merck filtrados a través de un filtro Millipore de $0,2 \mu \mathrm{m}$, degasificados y almacenados en atmósfera de Helio.

Patrones de lípidos Sigma Art. 178-1: Mezcla de colesterol, oleato de colesterol, oleato de metilo, ácido oleico y trioleína; en cantidades del $20 \%$ para cada componente.

Material y aparatos.

Cromatógrafo de líquidos Spectra-Physics compuesto por:

- Bomba 8700 XR para tres gradientes.

- Inyector Rheodyne de $10 \mu \mathrm{l}$.

- Detector UV/VIS de longitud de onda variable Spectra-Physics 8440 XR.

- Integrador Spectra-Physics 4290.

Columna Lịchrosorb Merck Si 60 de tamaño de partícula $7 \mu \mathrm{m}(25 \times 0,46 \mathrm{~cm})$.

Condiciones cromatográficas.

Como fase móvil se usa una mezcla de n-hexano2-propanol-ácido acético (100:0,5:0,1).
La detección se realiza a $206 \mathrm{~nm}$ y temperatura ambiente usando el siguiente gradiente de flujo:

$1-12$ minutos: $1,0 \mathrm{ml} / \mathrm{min} ; 13 \mathrm{~min}: 1,2 \mathrm{ml} / \mathrm{min} ; 16$ $\min : 1,5 \mathrm{ml} / \mathrm{min} ; 20-30 \mathrm{~min}: 1,7 \mathrm{ml} / \mathrm{min}$.

Tanto las muestras como los patrones se disuelven en el solvente de elución para disminuir al mínimo las señales del pico de solvente.

Se inyectan directamente en el cromatógrafo cantidades de $10 \mu \mathrm{g}$ de lípidos $/ 10 \mu \mathrm{l}$ de disolución.

Después de cada inyección se lava la columna con 20-25 $\mathrm{ml}$ de solventes de una serie de polaridad creciente: hexano, acetona y metanol. Diariamente se reactiva la columna con $30-40 \mathrm{ml}$ de cada uno de los solventes anteriores en orden inverso.

Identificación y comprobación de las fracciones.

La identificación de los componentes lipídicos se realiza por comparación y coincidencia de los tiempos de retención con los de una mezcla de patrones disueltos en la fase móvil.

Además como comprobación fueron colectadas las distintas fracciones separadas y desarrolladas en TLC de silicagel $G 60$ de $0,20 \mathrm{~mm}$ de espesor, previamente activadas durante una hora a $110^{\circ} \mathrm{C}$.

Como líquidos de desarrollo se emplean las siguientes mezclas en un desarrollo doble unidimensional:

a) Eter etílico-ácido acético $(50: 1)$ hasta $R_{f}=0,4$. 20:3)

b) Eter de petroleo-éter etílico-ácido acético (80:

\section{Precisión}

La precisión de los porcentajes de área para cada uno de los componentes lipídicos separados, ha sido estudiada efectuando 8 determinaciones sobre la misma muestra. Los coeficientes de variación se recogen en la Tabla I. La precisión del método es suficiente si se realiza la inyección por duplicado.

\section{RESULTADOS Y DISCUSION}

En la Figura 1 se presenta el cromatograma de una mezcla patrón compuesta por: oleato de colesterol, oleato de metilo, trioleína, ácido oleico y colesterol. El tiempo total de la determinación es de 26 minutos aproximadamente.

\section{Tabla 1}

Precisión (\% area) obtenida en la determinación de lípidos neutros del polen apícola comercial.

\begin{tabular}{lcccccc}
\hline & $\begin{array}{c}\text { Esteres de } \\
\text { esteroles }\end{array}$ & $\begin{array}{c}\text { Esteres de } \\
\text { acidos grasos }\end{array}$ & Triglicéridos & $\begin{array}{c}\text { Acidos } \\
\text { grasos }\end{array}$ & Colesterol \\
\hline$\overline{\mathbf{x}}$ & 47,0 & 9,1 & 8,1 & 6,9 & 27,2 \\
$\mathbf{B}_{\mathrm{n}-1}$ & 0,738 & 0,252 & 0,189 & 0,250 & 1,429 \\
Cv8 & 1,6 & 2,8 & 2,3 & 3,6 & 5,4
\end{tabular}




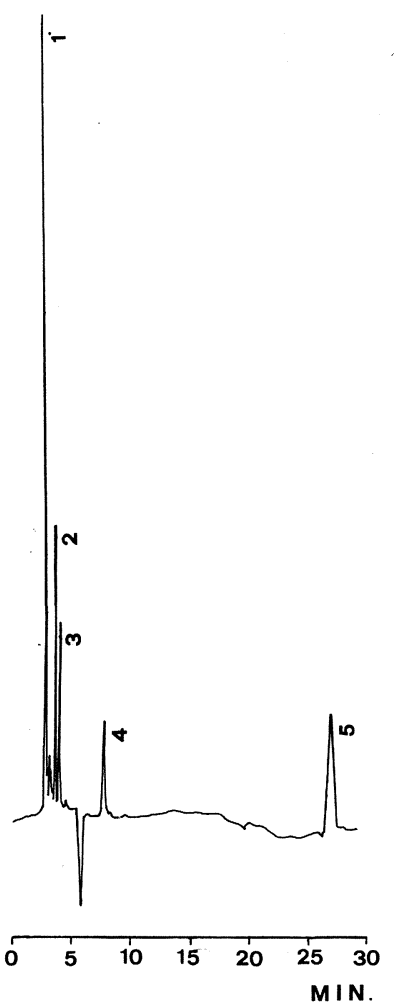

Figura 1

Cromatograma de una mezcla patrón de lípidos neutros: (1) Oleato de colesterol. (2) Oleato de metilo.

(3) Trileína. (4) Acido oleico. (5) Colesterol.

Cuando se compara la muestra (Figura 2) con la mezcla patrón, se puede observar que en las condiciones de trabajo empleadas, los ésteres de ácidos grasos y los esteroles no están presentes en cantidades detectables. Por lo tanto, en el caso de las 35 muestras analizadas, no sería necesaria la utilización del gradiente de flujo descrito, pudiéndose llevar a cabo la determinación en unos 8 minutos a flujo constante de $1 \mathrm{ml} / \mathrm{min}$.

En las muestras analizadas se han aislado tres fracciones:

- Componentes más apolares como ésteres de esteroles.

- Triglicéridos.

$\rightarrow$ Acidos grasos, que aparecen separados en dos picos según el grado de insaturación y/o el peso molecular.

La identificación de las distintas fracciones separadas por HPLC ha sido corroborada mediante TLC. En la Tabla II se recogen los valores de $R_{\text {f }}$ obtenidos para los componentes de la mezcla patrón, así como para las fracciones colectadas de la muestra.

En la primera fracción ( $T R=2,61$ ) aparece una coloración amarilla al concentrarla en la cromatoplaca, debida a la presencia de carotenoides. (Con

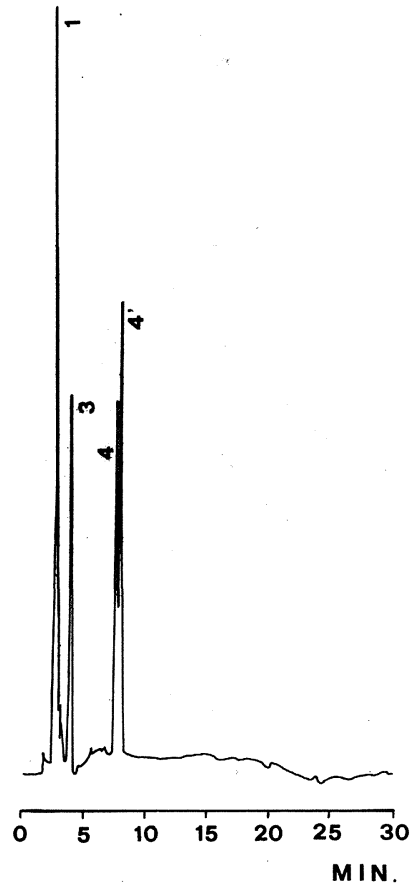

Figura 2

Cromatograma de las clases de lípidos neutros en una muestra de polen apícola: (1) Esteres de esteroles, carotenoides y ceras. (3) Triglicéridos. (4). Acidos grasos TR $=$ 7,42. (4') Acidos grasos $\mathrm{TR}=7,69$.

Tabla II

Valores de $R$ de las fracciones de lípidos neutros colectadas mediante HPLC y desarrolladas por TLC.

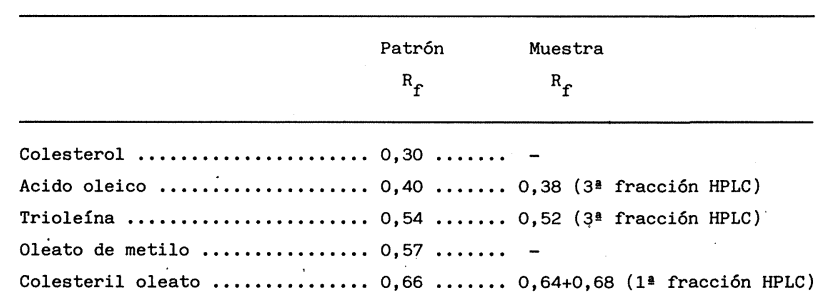

relación a estos compuestos está en preparación un estudio (14) donde se aisla y cuantifica la fracción de carotenos del polen apícola mediante HPLC). Al revelar las cromatoplacas con vapores de iodo se obtuvieron dos componentes en la primera fracción: uno coincide con el $R_{f}$ de los ésteres de esteroles del patrón y el otro corresponde al de las ceras. Por lo tanto, la primera fracción engloba también a los carotenoides y a las ceras.

Para los triglicéridos $(T R=3,77)$ se observa una clara coincidencia con el $R_{f}$ del patrón.

Con relación a los dos pícos que fueron separados mediante HPLC con tiempos de retención de 7,42 y 7,69, corresponden como se observa por TLC, a una única fracción de ácidos grasos. La composición 
de esta fracción ha sido determinada por GLC (12), siendo el ácido palmítico el ácido graso mayoritario con un 27,2 \%.

Así mismo, por TLC se ha verificado la ausencia de ésteres de ácidos grasos y esteroles en cantidades apreciables. Por otra parte, se ha determinado mediante GLC (11) los diferentes componentes de la fracción esterólica de las muestras de polen apícola, siendo el principal esterol el 5-avenasterol $(46,4 \%)$.

En la Tabla III se muestran los valores del porcentaje en área para cada uno de los componentes aislados en las muestras analizadas.

El componente mayoritario corresponde a la fracción de ésteres de esteroles y otros componentes apolares con un $40,6 \%$ de valor promedio, oscilando entre el $23,9 \%$ (muestra 21) y el $66,0 \%$ (muestra 8).

La proporción de ácidos grasos y triglicéridos en las muestras es muy similar, donde los primeros presentan un promedio total de 29,5 \% (11,0 + 18,5 $\%)$, oscilando entre el $19,0 \%$ en la muestra $7(9,9$ $+9,1 \%$ ) y el $46,1 \%$ en la muestra $19(14,2+31,9$ $\%)$. Por su parte los triglicéridos, tienen un valor promedio de $29,2 \%$, cuyos valores extremos son $9,9 \%$ (muestra 8) y $41,6 \%$ (muestra 18), respectivamente.

\section{Tabla III}

Contenido (\% área) de lípidos neutros de las muestras de polen apícola analizadas.

\begin{tabular}{|c|c|c|c|c|}
\hline \multirow{2}{*}{$\begin{array}{l}\text { Ne de } \\
\text { moestra }\end{array}$} & \multirow{2}{*}{$\begin{array}{c}\text { Esteres de sateroles } \\
\text { carotenoides } \\
\text { ceras } \\
T R=2,61\end{array}$} & \multirow{2}{*}{$\begin{array}{l}\text { Trigliceridos } \\
\text { TRe3,T }\end{array}$} & \multicolumn{2}{|c|}{ Acidos grasos } \\
\hline & & & $T R=7,42$ & $T R=7,69$ \\
\hline 1 & 56,2 & 22,8 & 9,1 & 11,6 \\
\hline 2 & 36,3 & 20,7 & 14,1 & 28,2 \\
\hline 3 & 54,6 & 21,4 & 3,0 & 20,7 \\
\hline 4 & 35,5 & 18,9 & 15,1 & 30,3 \\
\hline 5 & 46,3 & 36,1 & 10,2 & 6,7 \\
\hline 6 & 51,5 & 27,0 & 9,0 & 12,4 \\
\hline 7 & 45,0 & 33,0 & 9,9 & 9,1 \\
\hline 8 & 66,0 & 9,9 & 11,6 & 12,1 \\
\hline 9 & 41,4 & 33,0 & 7,1 & 12,8 \\
\hline 10 & $37,9$. & 32,5 & 10,2 & 18,6 \\
\hline 11 & 31,6 & 29,8 & 14,9 & 23,1 \\
\hline 12 & 41,4 & 31,4 & 11,7 & 15,2 \\
\hline 13 & 52,8 & 24,3 & 5,1 & 17,8 \\
\hline 14 & 4,5 & 22,4 & 11,3 & 21,8 \\
\hline 15 & 38,3 & 33,4 & 11,1 & 17,0 \\
\hline 16 & 38,5 & 33,6 & 11,2 & 17,2 \\
\hline 17 & 40,0 & 36,3 & 9,1 & 14,4 \\
\hline 18 & 24,5 & 41,6 & 12,3 & 21,4 \\
\hline 19 & 23,9 & 29,5 & 14,2 & 31,9 \\
\hline 20 & 38,5 & 38,2 & 8,8 & 14,1 \\
\hline 21 & 23,9 & 37,4 & 13,5 & 24,7 \\
\hline 22 & 42,4 & 28,7 & 10,0 & 18,4 \\
\hline 23 & 35,3 & 28,2 & 9,6 & 25,6 \\
\hline 24 & 38,4 & 22,0 & 19,3 & 19,7 \\
\hline 25 & 54,8 & 21,8 & 10,3 & 12,7 \\
\hline 26 & 43,1 & 36,9 & 11,3 & 8,4 \\
\hline 27 & 27,6 & 39,6 & 13,8 & 18,5 \\
\hline 28 & 44,6 & 23,6 & 14,6 & 16,5 \\
\hline 29 & 45,8 & 20,1 & 6,5 & 27,2 \\
\hline 30 & 38,3 & 30,0 & 11,9 & 17,6 \\
\hline 31 & 32,4 & 31,1 & 16,2 & 19,6 \\
\hline & 37,6 & 26,1 & 12,5 & 23,0 \\
\hline 33 & 34,3 & 37,6 & 14,3 & 13,2 \\
\hline 34 & 40,7 & 22,7 & 1,8 & 34,6 \\
\hline 35 & 37,4 & 41,0 & 10,3 & 11,1 \\
\hline$\overline{\mathbf{x}}$ & 40,6 & 29,2 & 11,0 & 18,49 \\
\hline & 9,35 & 7,41 & 3,56 & 6,79 \\
\hline Minfino $(x)$ & 23,9 & 9,9 & 1,8 & 6,7 \\
\hline Maximo $(x)$ & 66,0 & 41,6 & 19,3 & 34,6 \\
\hline
\end{tabular}

\section{CONCLUSIONES.}

Es interesante el método de HPLC descrito para la separación de lípidos neutros del polen apícola, dadas las escasas referencias bibliográficas sobre estos parámetros en este alimento. Además destaca la rapidez de la determinación, ya que en el caso de las muestras analizadas es de unos 8 minutos.

Se comprueba que inyectando la muestra directamente sin realizar una separación previa de los componentes polares, se mantiene la eficacia de la columna con lavados y reactivaciones frecuentes.

\section{AGRADECIMIENTO}

A la Consellería de Eduación de la Xunta de Galicia por la concesión de la Beca de Investigación a D. a Soledad Muniategui durante el curso 1988-1989.

\section{BIBLIOGRAFIA}

1. Andrikopoulos, N. K.; Siafak-Kapadai, A.; Demopoulos, C. A. and Kapoulas, V. M.- "Lipids of Pinus halepensis poIlen".- Phytochemistry 24 (1985) 2953-2957.

2. Fricke, H. S. G. and Oehlenschläger, J.- "Separation of lipids from Antartic Krill (Euphausia Superba Dana) by isocratic high-performance liquid chromatography on silica using flow-program".- J. Chromatogr. 252 (1982) 331-334.

3. Hamilton, F, G. and Conai, K.- "Separation of neutral lipids and free fatty acids by high-performance liquid chromatography using low warelength ultraviolet detection".- J. Lipid Res. 25 (1984) 1142-1148.

4. Graille, J., Frater, C, and Pioch, D.- "HPLC analysis of glicerides in lipolysis and alcoholysis reaction".- Fat Science $16^{\text {th }}$ ISF Congress., Budapest, (1983) 79-90.

5. Lapin, B. P., Piseva, N. A., Rubtsova, T. E. and lakevich, M. L.- "Separation of lipophilic fractions by high-performance liquid chromatography".- J. Chromatogr. 365 (1986) 229 235.

6. Greenspan, M. D, and Schroeder, E. A.- "Separation and detection of neutral lipids and free fatty acids in a liver extrat by highperformance liquid chromatography".-Anal. Biochem. 127 (1982) 441-448.

7. Ritchie, J. and Jee, F.- "High-performance liquid chromatographic techniques for the separation of lipids classes".J. Chromatogr. 329 (1985) 273-280.

8. Christie, W. W.- "Separation of lipid classes by high-performance liquid chromatography whit the mass detector".J. Chromatogr 361 (1986) 396-399.

9. Aitzetmüller, K.- "High Performance Liquid Chromato-graphic analysis of partial glycerides and other technical lipid mixtures".- J. Chromatogr. 139 (1977) 61-68.

10. Aitzetmüller, K. and Koch, J.- "Liquid chromatographic analysis of sebum lipids and other lipids of medical interest".- J. Chromatogr. 145 (1978) 195-202.

11. Simal, J., Huidobro, J. F. y Muniategui, S.- "Estudio de la fracción esterólica del polen apicola".- Grasas y Aceites 39 (1988) 327-333

12. Muniategui, S., Simal, J., Huidobro, J. F. y García. M."Estudio de los ácidos grasos del polen apícola".- Grasas y Aceites 40 (1989) 81-86.

13. UNA NORMA ESPAÑOLA, UNE 34.082,76.- Determinación de la grasa total en chocolates.- Instituto de Racionalización del Trabajo.

14. Muniategui, S., Sancho, M. T., López, J., Huidobro, J. F. y Simal, J.- "Determinación de carotenos en el polen apícola mediante cromatografía líquida de alta resolución".- (En preparación).

(Recibido: Octubre 1990) 